\title{
Simulation des Grandes Échelles d'écoulements turbulents compressibles dans des conduits courbes : étude des transferts thermiques
}

\author{
Cécile Münch ${ }^{\mathrm{a}}$ et Olivier Métais \\ L.E.G.I./MOST, BP 53, 38041 Grenoble Cedex 09, France
}

Reçu le 25 février 2005, accepté le 21 mars 2005

\begin{abstract}
Résumé - Nous présentons des Simulations des Grandes Échelles d'écoulements turbulents compressibles tridimensionnels se développant dans des conduits courbes de section rectangulaire. Ces écoulements sont caractérisés par la présence de flux secondaires intenses et de structures turbulentes responsables des transferts de masse et thermique. Le but est ici d'évaluer l'influence du rapport d'aspect de la section sur ce type d'écoulements. Les résultats montrent que ce paramètre modifie l'intensité et la localisation des tourbillons et donc indirectement le transfert de chaleur lorsqu'un chauffage est appliqué sur la paroi convexe.
\end{abstract}

Mots clés : Turbulence / échange thermique / simulation numérique

\begin{abstract}
Large Eddy Simulations of compressible turbulent flows in curved ducts with heat transfer. Large Eddy Simulations of three-dimensional turbulent compressible flows in curved duct of rectangular cross-section are presented. Strong secondary flows and turbulent structures are the typical features of this type of flow: these are responsible for most of the heat and mass transfer within the duct. Our goal is here to investigate the influence of the duct aspect ratio on the flow development. The results show that this parameter modifies the flow structures in terms of intensity and localisation. Thus it significantly influences the heat transfers when a higher temperature is imposed on the duct convex wall.
\end{abstract}

Key words: Turbulence / heat transfer / numerical simulation

\section{Introduction}

Les écoulements dans les canaux courbes sont présents dans de nombreuses applications industrielles comme les turbines, les échangeurs de chaleur ou les canaux de refroidissement des moteurs de fusée. De nombreuses études expérimentales ont été menées pour caractériser ces écoulements complexes. Elles révèlent l'existence de flux secondaires intenses qui se développent en une paire de tourbillons contra-rotatifs $[1,2]$. Lorsque le conduit présente une section avec un rapport d'aspect l'interaction entre ces deux tourbillons est modifiée [3]. Numériquement, les études des écoulements turbulents en conduits courbes sont plus rares du fait de la difficulté de reproduire les flux secondaires ainsi que l'instabilité centrifuge naissant sur la paroi concave, [4]. Nous montrons ici que la Simulation des Grandes Échelles constitue un outil efficace pour reproduire correctement ces instabilités

a Auteur correspondant : cecile.munch@hmg.inpg.fr et les flux secondaires associés en particulier dans les conduits chauffés [5].

\section{Méthologie numérique}

Les Simulations des Grandes Échelles (SGE) sont basées sur les équations de Navier-Stockes tridimensionnelles compressibles filtrées à l'aide d'un filtre passe-bas et écrites sous forme conservative. L'action de la turbulence sous-maille sur l'écoulement à grande échelle est prise en compte grâce à une viscosité turbulente basée sur la fonction de structure sélective [6]. Le système est complété par trois équations : la loi de Sutherland décrivant la variation de la viscosité avec la température, la loi des gaz parfaits et le nombre de Prandtl turbulent considéré comme constant égal à 0,6 . Le schéma numérique utilisé pour discrétiser ces équations est le schéma compact de McCormack d'ordre 4 en espace et 2 en temps [7]. La géométrie du domaine de calcul est un conduit de section 


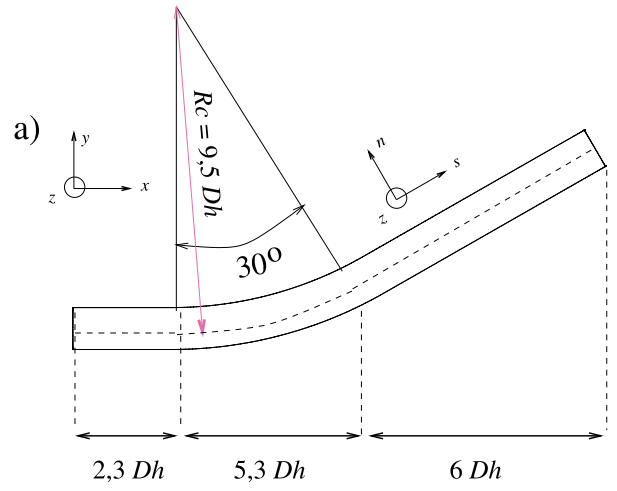

b)

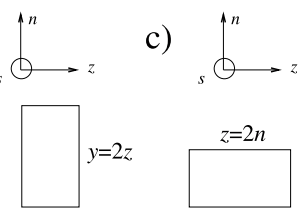

Fig. 1. Géométrie des domaines de calcul.

Tableau 1. Caractéristiques des domaines de calcul.

\begin{tabular}{llll}
\hline section & carrée & rectangulaire $2: 1$ (haute) & rectangulaire 1:2 (large) \\
Taille du domaine & $13,6 D_{\mathrm{h}} \times D_{\mathrm{h}} \times D_{\mathrm{h}}$ & $13,6 D_{\mathrm{h}} \times 1,5 D_{\mathrm{h}} \times 0,75 D_{\mathrm{h}}$ & $13,6 D_{\mathrm{h}} \times 0,75 D_{\mathrm{h}} \times 1,5 D_{\mathrm{h}}$ \\
Résolution & $160 \times 50 \times 50$ & $160 \times 100 \times 50$ & $160 \times 50 \times 100$ \\
\hline
\end{tabular}

rectangulaire de diamètre hydraulique $D_{\mathrm{h}}$ et de longueur totale approximativement égale à $14 D_{\mathrm{h}}$. La courbure est définie par un angle de 30 degrés et un rayon de courbure moyen égal à $9,5 D_{\mathrm{h}}$. La section droite d'entrée a une longueur de $2,3 D_{\mathrm{h}}$, celle de sortie une longueur $6 \mathrm{Dh}$ (cf. Fig. 1a). Trois configurations sont considérées : un conduit avec une section carrée, deux conduits de section rectangulaire de rapport d'aspect 2:1 (conduit haut) ou 1:2 (conduit large) (voir Figs. 1b et c respectivement). Le maillage utilisé dans ces domaines est orthogonal non uniforme avec un nombre de points donné dans le tableau 1. Dans les deux directions perpendiculaires à l'écoulement principal, une loi en tangente hyperbolique est appliquée au maillage de sorte que $n_{\min }^{+}=z_{\min }^{+}=1,8$. Suivant l'abscisse curviligne $s$, un resserrement des mailles est appliqué en sortie de conduit. L'originalité de notre calcul réside dans le fait que l'écoulement en entrée du conduit courbe est pleinement développé, il évolue ensuite spatialement. Pour obtenir une telle condition d'entrée, un écoulement dans un conduit périodique en amont est calculé simultanément et fournit un champ d'entrée au conduit spatial à chaque pas de temps. Sur les parois, la vitesse est nulle et la température uniforme. Pour les conditions limites d'entrée et de sortie du conduit spatial, nous utilisons la méthode des caractéristiques [8]. Les paramètres de l'écoulement pour les trois simulations sont : le nombre de Reynolds basé sur la vitesse débitante et $D_{\mathrm{h}}$ égal à 6000 , le nombre de Mach égal à 0,5 et le nombre de Prandtl égal à 0,7. Les effets de gravité sont négligeables dans la gamme de paramètres considérée ici.

\section{Résultats et discussion}

\subsection{Conduits non-chauffés}

Dans un premier temps, nous allons étudier l'influence sur l'écoulement de la distance entre les deux parois courbes du conduit. Tirant parti de la symétrie des quantités moyennes par rapport au plan médian du conduit, les figures $2 \mathrm{a}-\mathrm{c}$ montrent des demi-sections au niveau de la sortie $\left(s / D_{\mathrm{h}}=13\right)$ des trois conduits. Sur la gauche de chaque figure sont représentés les flux secondaires (perpendiculaires au flux principal) moyennés en temps. Pour des raisons de visibilité, seul un vecteur sur deux est représenté. Les écoulements en conduit courbe se caractérisent par la présence de flux secondaires dits du premier type de Prandtl. Leur intensité dépend essentiellement du rayon de courbure, dans notre cas, ils atteignent environ $20 \%$ de la vitesse débitante à la sortie de la partie courbe. Deux cellules contra-rotatives de type Ekman apparaissent de chaque côté du plan de symétrie près de la paroi convexe située dans la partie supérieure de chaque figure [9]. En sortie des conduits, l'intensité des flux secondaires n'atteint plus que $10 \%$ de la vitesse débitante. Dans le cas du conduit carré, le maximum d'intensité des flux secondaires se situe dans le plan de symétrie (cf. Fig. 2a). En effet, les deux cellules sont suffisamment proches en sortie pour générer un courant descendant important dirigé de la paroi convexe vers la paroi concave au niveau du plan de symétrie. Dans le cas du conduit haut, on observe le même phénomène (cf. Fig. 2c). La cellule est encore plus étirée dans la direction $\vec{n}$. Lorsque le conduit est large, les cellules ne parviennent pas à occuper toute la largeur (cf. Fig. 2b). Elles interagissent plus faiblement et le maximum des flux secondaires se situe alors près de la paroi convexe. À droite de chaque figure sont représentées des iso-valeurs de la vorticité longitudinale, $\omega_{\mathrm{s}}$, avec un pas de 0,3 . Cette représentation permet d'observer plus précisément la forme et la localisation des cellules contra-rotatives. Pour les conduits carrés et larges, la valeur de $\omega_{\mathrm{s}}$ au cour de la cellule est de $-0,68 U_{\mathrm{b}} / D_{\mathrm{h}}$. Par contre dans le cas du conduit haut (cf. Fig. 2c droite), le minimum de $\omega_{\mathrm{s}}$ est de $-0,48 U_{\mathrm{b}} / D_{\mathrm{h}}$ au centre du tourbillon. Les structures de type Ekman ont moins de place pour se développer, et leur intensité est plus faible que dans les deux autres cas. 


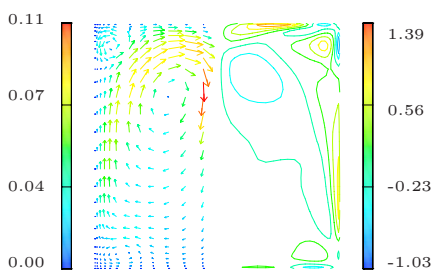

(a)

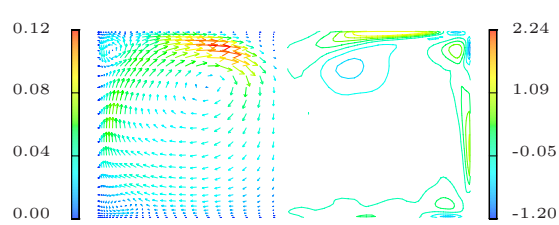

(b)

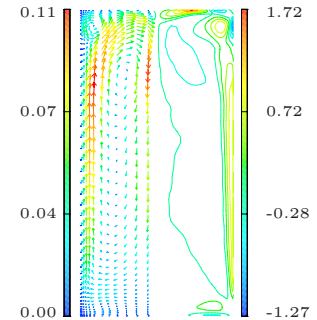

(c)

Fig. 2. Flux secondaires et iso-valeurs de la vorticité longitudinale [pas : 0,3] en sortie des trois conduits.

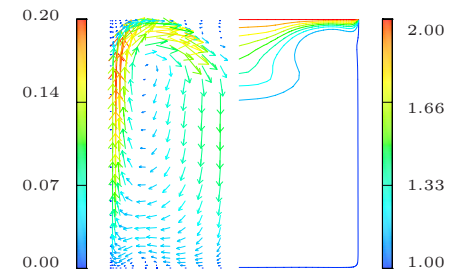

(a)

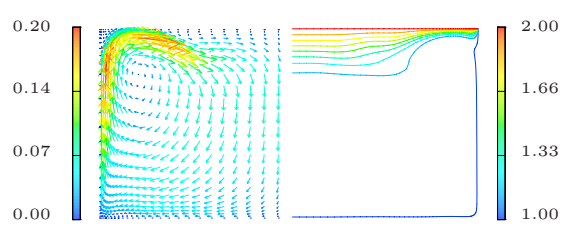

(b)

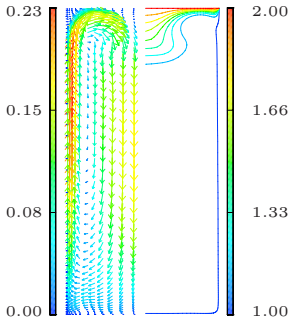

(c)

Fig. 3. Flux secondaires et iso-valeurs de la température [pas : 0,1] en sortie de la courbure.

\subsection{Conduits chauffés}

Dans cette partie, nous allons évaluer l'influence du rapport d'aspect du conduit sur le transfert de chaleur. Pour cela, nous appliquons une température supérieure sur la paroi convexe, égale à deux fois la température sur les trois autres parois. Sur la figure 3, nous avons représenté des coupes des flux secondaires moyens et des iso-valeurs de la température moyenne en sortie de la partie courbe, en $s / D_{\mathrm{h}}=7,6$, dans les trois conduits. C'est dans cette partie du conduit que les flux secondaires sont les plus intenses. Ils transportent le fluide chaud et lent de la paroi convexe vers le cour de l'écoulement dans la zone du plan de symétrie. Dans cette région, les iso-lignes de température sont de ce fait très distantes les unes des autres. Plus près des parois latérales, les flux secondaires apportent du fluide froid rapide des parois planes vers la paroi convexe. La température varie donc très rapidement dans cette zone. Lorsque le conduit est carré ou haut (cf. Figs. 3a et c) la forme des iso-valeurs de la température est très similaire. Par contre les flux secondaires sont plus intenses dans le conduit rectangulaire : $23 \%$ de la vitesse débitante. Dans le conduit large (cf. Fig. 3b), les flux secondaires sont dirigés de la paroi convexe vers la paroi concave dans une plus large zone. Cela se traduit par une plus large propagation du fluide chaud entre les deux parois latérales.

Afin de décrire le transfert de chaleur de manière plus précise, nous avons calculé le flux de chaleur sur la paroi convexe : $Q(s, z)=\left.\kappa \frac{\partial T}{\partial n}\right|_{\text {paroi }}$ avec $\kappa$ la conductivité thermique. Ce flux est normalisé par la température sur les parois non-chauffées et le diamètre hydraulique. Sur les figures $4 \mathrm{a}$ et b, $Q$ est représenté en fonction de l'abscisse curviligne $s$, dans le plan de symétrie et dans le plan du quart de la paroi convexe, c'est-à-dire à $\frac{z}{D_{\mathrm{h}}}=0,25$ dans le cas du conduit carré, à $\frac{z}{D_{\mathrm{h}}}=0,1875$ dans le conduit haut et à $\frac{z}{D_{\mathrm{h}}}=0,375$ pour le conduit large. Les lignes verticales symbolisent le début et la fin de la partie courbe. En entrée des trois conduits avant la courbure, le comportement des différentes courbes est similaire dans les trois cas et ceci pour les deux plans $z$ considérés. Le flux de chaleur diminue du fait du développement de la couche limite thermique et donc de l'atténuation du gradient de température normal à la paroi. Dans la partie courbe, le flux de chaleur continue de diminuer dans le plan de symétrie : ceci est dû au transport du fluide chaud vers le cœur du conduit par l'éjection se formant sur la paroi convexe. Lorsque le conduit est haut (- - $)$, les flux secondaires étant plus intenses, l'éjection est plus forte et le flux de chaleur plus faible. Au contraire, dans le conduit large $(\cdots \cdots)$, les cellules de Ekman étant plus éloignées l'une de l'autre l'éjection est plus faible et le flux de chaleur diminue moins rapidement. La différence la plus importante se situe au quart du conduit (4b). En effet, le flux de chaleur est dix fois plus important que dans le plan de symétrie dans les cas des conduits carré et haut. Cette augmentation est due à la présence des cellules d'Ekman qui apportent du fluide froid au voisinage de la paroi convexe (cf. Fig. 3). Par contre, dans le cas du conduit large, les cellules d'Ekman ne se situent pas à cet endroit mais sont plus proches des parois latérales. Il n'y a donc pas d'apport de fluide froid et le flux de chaleur se comporte comme dans le plan de symétrie. En sortie de conduit, l'effet de courbure disparaît. Les cellules s'éloignent des parois latérales et de la paroi convexe (cf. Fig. 2), de plus l'intensité des flux secondaires diminue. Pour le flux de chaleur, cela se traduit par une faible augmentation dans le plan de symétrie car l'éjection est moins 


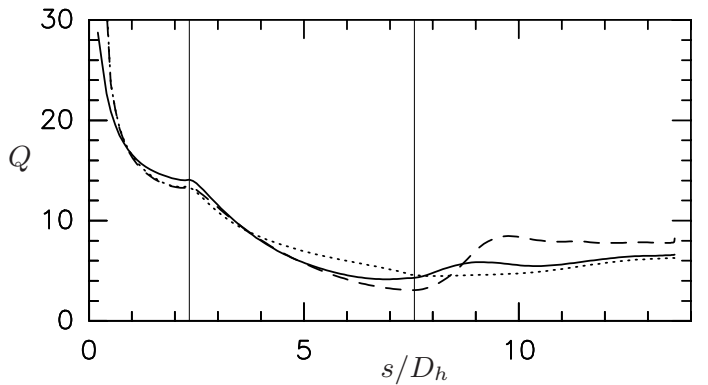

(a)

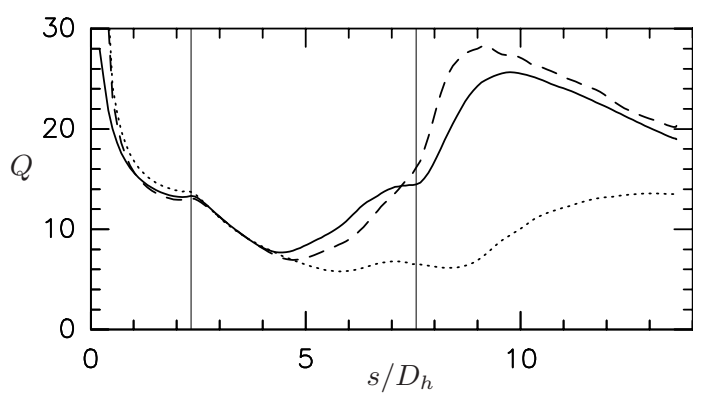

(b)

Fig. 4. Flux de chaleur dans le conduit carré ( - ), large $(\cdots \cdot)$, haut $(---)$ : a) dans le plan de symétrie, b) au quart de la paroi convexe.

intense pour les trois cas de rapport d'aspect. Dans l'autre plan $z$ des conduits carré et haut, $Q$ diminue car les cellules se sont éloignées de la paroi convexe. Au contraire dans le cas du conduit large, le flux de chaleur augmente car les cellules d'Ekman sont alors plus proches du plan de symétrie (cf. Fig. 2b) et les flux secondaires apportent maintenant du fluide froid vers la paroi convexe.

\section{Conclusions}

Cette étude numérique menée en utilisant la Simulation des Grandes Échelles montre l'influence du rapport d'aspect du conduit sur l'écoulement. Les structures s'adaptent à l'espace qui leur est donné. Dans le cas du conduit large, l'interaction entre les deux cellules est plus faible que dans le cas du conduit haut car elles sont plus éloignées l'une de l'autre. Ceci modifie l'intensité du flux secondaire dirigé de la paroi convexe vers la paroi concave dans le plan de symétrie. Dans le cas où un chauffage est appliqué sur la paroi convexe, cela se traduit par une éjection du fluide chaud vers le cœur de l'écoulement moins intense pour le conduit large mais plus étendue entre les deux parois latérales. Le flux de chaleur est ainsi plus homogène dans la direction transverse dans le conduit large.

Remerciements. Certains calculs ont été effectués à l'IDRIS (Institut du Développement et des Ressources en Informatique Scientifique). Ce travail est soutenu par le CNES (Centre National d'Études Spatiales).

\section{Références}

[1] J.A.C. Humphrey, J.H. Whitelaw, G. Yee, Turbulent flow in a square duct with strong curvature, J. Fluid Mechanics 103 (1981) 443-463

[2] S.M. Chang, J.A.C. Humphrey, A. Modavi, Turbulent flow in a strongly curved U-bend and Downstream tangent of square cross-sections, PhysicoChemical Hydrodynamics 4(3) (1983) 243-269

[3] W.J. Kim, V.C. Patel, Origin and decay of longitudinal vortices in developing flow in a curved rectangular duct, J. Fluids Engineering 116 (1994) 45-52

[4] Y.D. Choi, H. Iacovides, B.E. Launder, Numerical computation of turbulent flow in a square sectioned $180 \mathrm{Deg}$ bend, J. Fluids Engineering 111 (1989) 59-68

[5] M. Salinas Vazquez, O. Métais, Large-Eddy Simulation of the turbulent flow through a heated square duct, J. Fluid Mechanics 453 (2002) 201-238

[6] M. Lesieur, O. Métais, New trends in Large Eddy Simulations of turbulence, Ann. Rev. Fluid Mech. 28, (1996) 45-82

[7] C.A. Kennedy, M.H. Carpenter, Comparaison of several numerical Methods for simulation of compressible shear layers, NASA technical paper, Paper 3484, (1997)

[8] T. Poinsot, S. Lele, Boundary conditions for direct simulations of compressible viscous flows, J. Computational Physics 101 (1992) 104-129

[9] C. Münch, J. Hébrard, O. Métais, Large-eddy simulation of turbulent flow in curved and S-shape ducts, Direct and Large Eddy Simulation V, R. Friedrich, B. Geurts, O. Métais (eds.), ercoftac Kluwer, 2004, 527-536 